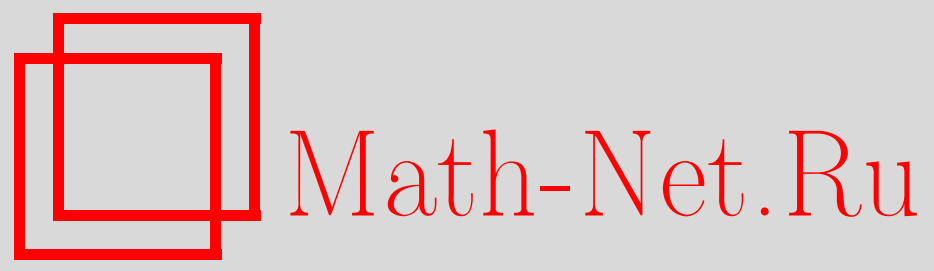

В. С. Рублев, Н. Б. Чаплыгина, Выбор критерия оптимизации в задаче о равномерном назначении, Дискрет. матем., 2005, том 17, выпуск 4, 150-157

DOI: https://doi.org/10.4213/dm137

Использование Общероссийского математического портала Math-Net.Ru подразумевает, что вы прочитали и согласны с пользовательским соглашением http://www . mathnet.ru/rus/agreement

Параметры загрузки:

IP : 54.224 .187 .69

26 апреля 2023 г., 13:16:09

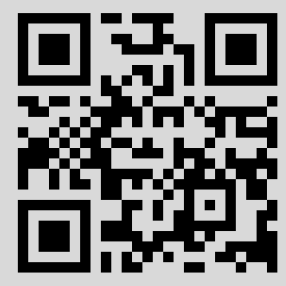




\title{
Выбор критерия оптимизации в задаче о равномерном назначении
}

\author{
(c) 2005 г. В. С. Рублев, Н. Б. Чаплыгина
}

\begin{abstract}
Во многих задачах оптимизационного характера определяется критерий оптимизации, в качестве которого может использоваться числовой функционал, требующий максимизации или минимизации своего значения. Нередко возникает ситуация, когда на роль критерия может претендовать не один функционал, а несколько. Как правило, выбор критерия происходит согласно некоторым интуитивным соображениям и влияет на нахождение решения задачи. Рассматривая задачу с различными критериями, можно получить различные ее решения и поэтому приходится находить дополнительную информацию и проводить дополнительные исследования для наиболее приемлемого выбора критерия оптимизации.

В задаче о равномерном назначении в качестве критерия равномерности может быть принят любой симметрический функционал, обладающий свойствами нормы. Однако, несмотря на столь большой выбор, решения, которые минимизируют один из критериев, квадратичное отклонение числа работ каждого работника от среднего, оказываются минимизирующими и все остальные возможные критерии, что решает вопрос о выборе критерия оптимизации.
\end{abstract}

\section{1. Постановка задачи о равномерном назначении}

Кратко опишем постановку задачи о равномерном назначении, данную в [3]. В течение планируемого периода из $m$ дней $n$ работников выполняют определенный объем работ. В каждый $k$-й день, $k \in\{1, \ldots, m\}$, они должны произвести $s_{k}$ различных работ. Общее число работ равно $s=s_{1}+s_{2}+\ldots+s_{m}$. Возможности выполнения работ заданы матрищей

$$
R=\left\{r_{i j}^{k}, i \in\{1, \ldots, n\}, k \in\{1, \ldots, m\}, j \in\left\{1, \ldots, s_{k}\right\}\right\}
$$

где

$$
r_{i j}^{k}= \begin{cases}1, & \text { если } i \text {-й работник в } k \text {-й день может выполнить } j \text {-ю работу, } \\ 0, & \text { если } i \text {-й работник не имеет такой возможности. }\end{cases}
$$

При этом должны быть выполнены условия корректности задачи: назначения на каждый день возможны.

Необходимо равномерно распределить работы между работниками, учитывая возможность задания обязательных назначений при помощи булевой матрищы

$$
A=\left\{a_{i j}^{k}, i \in\{1, \ldots, n\}, k \in\{1, \ldots, m\}, j \in\left\{1, \ldots, s_{k}\right\}\right\}
$$


построенной аналогично матрище $\boldsymbol{R}$. При этом должны выполнятъся условия

$$
\begin{aligned}
a_{i j}^{k} \leqslant r_{i j}^{k}, & i \in\{1, \ldots, n\}, \quad k \in\{1, \ldots, m\}, \quad j \in\left\{1, \ldots, s_{k}\right\}, \\
\sum_{i=1}^{n} a_{i j}^{k} \leqslant 1, \quad k \in\{1, \ldots, m\}, \quad j \in\left\{1, \ldots, s_{k}\right\}, & \\
\sum_{j=1}^{s_{k}} a_{i j}^{k} \leqslant 1, & i \in\{1, \ldots, n\}, \quad k \in\{1, \ldots, m\} .
\end{aligned}
$$

Эту задачу будем называть $A$-задачей.

Допустимым решением $\boldsymbol{A}$-задачи назовем матрицу

$$
X=\left\{x_{i j}^{k}, i \in\{1, \ldots, n\}, k \in\{1, \ldots, m\}, j \in\left\{1, \ldots, s_{k}\right\}\right\},
$$

элементы которой удовлетворяют условиям

$$
0 \leqslant a_{i j}^{k} \leqslant x_{i j}^{k} \leqslant r_{i j}^{k} \leqslant 1, \quad i \in\{1, \ldots, n\}, \quad k \in\{1, \ldots, m\}, \quad j \in\left\{1, \ldots, s_{k}\right\},
$$

каждая работа должна быть выполнена:

$$
\sum_{i=1}^{n} x_{i j}^{k}=1, \quad k \in\{1, \ldots, m\}, \quad j \in\left\{1, \ldots, s_{k}\right\},
$$

и, как следствие,

$$
\sum_{i, k, j} x_{i j}^{k}=s,
$$

каждый рабочий выполняет не более одной работы в день:

$$
\sum_{j=1}^{s_{k}} x_{i j}^{k} \leqslant 1, \quad i \in\{1, \ldots, n\}, \quad k \in\{1, \ldots, m\} .
$$

Допустимому решению $X$ сопоставим итоговый вектор $X=\left(x_{1}, \ldots, x_{n}\right)$ (обозначив его для простоты тем же символом) числа работ каждого работника, где

$$
x_{i}=\sum_{k=1}^{m} \sum_{j=1}^{s_{k}} x_{i j}^{k}, \quad i=1, \ldots, n
$$

Поскольку каждая работа должна быть выполнена, сумма $x_{1}+x_{2}+\ldots+x_{n}$ равна общему числу работ $s$. Заметим, что задача в такой постановке не предполагает необходимость существования допустимых решений.

Оптимальным решением $A$-задачи назовем допустимое решение, минимизирующее функщионал

$$
L(X)=\sum_{i=1}^{n}\left(x_{i}-\bar{s}\right)^{2},
$$

где $\bar{s}$ - среднее число работ на каждого рабочего,

$$
\bar{s}=\frac{1}{n} \sum_{i=1}^{m} s_{i} .
$$


Критерий $L(X)$ равносилен в данном случае средне квадратичному отклонению

$$
\sqrt{\sum_{i=1}^{n}\left(x_{i}-\bar{s}\right)^{2}}
$$

В качестве критериев равномерности наряду с введенным могут быть использованы критерии

$$
\begin{aligned}
& f_{1}(X)=\max _{1 \leqslant i \leqslant n} x_{i}, \\
& f_{2}(X)=\max _{1 \leqslant i \leqslant n}\left|x_{i}-\bar{s}\right|, \\
& f_{3}(X)=\frac{1}{n} \sum_{i=1}^{n}\left|x_{i}-\bar{s}\right|,
\end{aligned}
$$

Оказывается, что функционал $L$ является наиболее сильным не только из представленных выше, но и из всех симметрических, удовлетворяющих свойствам нормы критериев, то есть решения, оптимизирующие критерий $L(X)$, являются оптимальными и для любого другого критерия, определенного симметрическим однородным функционалом. Прежде, чем это доказывать, приведем свойства решения, оптимального относительно функционала-критерия $L$, полученные в [3]. Именно такое решение и будем далее называть $L$-оптимальным или просто оптимальным решением $A$-задачи.

\section{2. Постановка $A l$-задачи и характеристическое свойство оптимального решения}

Для установления характеристического свойства оптимального решения $A$-задачи вводится вспомогательная $A l$-задача о максимальных назначениях при ограничении на число работ каждого работника.

Пусть для некоторого плана работ заданы матрица $R$ возможностей каждого работника, матрица $A$ обязательных назначений, удовлетворяющие условиям (1)-(3), и некоторое целое число $l>0$, которое ограничивает сверху итоговое число работ каждого работника (в случае, когда есть обязательные назначения, оно может нарушаться).

Назовем решением, допустимым для $A l$-задачи, матрицу назначений $X$, удовлетворяющую условиям (4) и (5) и ограничению

$$
\sum_{i=1}^{n} x_{i j}^{k} \leqslant 1, \quad k \in\{1, \ldots, m\}, \quad j \in\left\{1, \ldots, s_{k}\right\},
$$

причем такую, что для всех $i \in\{1, \ldots, n\}$

$$
\sum_{k=1}^{m} \sum_{j=1}^{s_{k}} a_{i j}^{k} \leqslant x_{i}=\sum_{k=1}^{m} \sum_{j=1}^{s_{k}} x_{i j}^{k} \leqslant \max \left\{\sum_{k=1}^{m} \sum_{j=1}^{s_{k}} a_{i j}^{k}, l\right\} .
$$

Эти условия означают, что каждый работник, во-первых, имеет все обязательные для него назначения и, во-вторых, если их число меньше $l$, может иметь некоторые дополнительные назначения, число которых не превышает разности между $l$ и числом обязательных 
назначений. Назовем оптимальным решением $A l$-задачи допустимое для этой задачи решение $X$, максимизирующее общее число выполненных работ:

$$
\varphi(X)=\sum_{i=1}^{n} x_{i}=\sum_{i=1}^{n} \sum_{k=1}^{m} \sum_{j=1}^{s_{k}} x_{i j}^{k} \rightarrow \max .
$$

Для любого натурального $l$ назовем $l$-срезкой матрицы $X$, являющейся решением $A$-задачи, матрицу $X[l]$, полученную следующим образом:

(a) $X[l]$ содержит все единичные (равные 1) элементы матрицы $A$,

(б) в каждой $i$-й строке матрицы $X$, содержащей более, чем $\max \left\{a_{i}, l\right\}$ единичных элементов, оставлены равными 1 ровно $\max \left\{a_{i}, l\right\}$ элементов (любых, но включая все обязательные назначения), а остальные заменены нулем ( $l$-срезка может быть не единственной для матрицы $X$ ),

(в) все остальные строки матрицы $X$ оставлены без изменения.

Поскольку

$$
a_{i j}^{k} \leqslant x[l]_{i j}^{k} \leqslant x_{i j}^{k}
$$

To

$$
x[l]_{i}= \begin{cases}x_{i}, & \text { если } x_{i}=a_{i} \text { или } x_{i} \leqslant l, \\ l, & \text { если } x_{i} \neq a_{i} \text { и } x_{i}>l .\end{cases}
$$

Справедлива следующая доказанная в [3] теорема о характеристическом свойстве оптимального решения.

Теорема 1. Для $A$-задачи допустимое решение $X$ оптимально тогда и только тогда, когда для любого $l>0$ каждая l-срезка $X$ явяется оптимальным решением $A l$-задачи.

\section{3. Оптимальный критерий равномерности}

Лемма 1. Если $X=\left(x_{1}, x_{2}, \ldots, x_{n}\right)$ - упорядоченный по неубыванию координат итоговый вектор L-оптимального решения, $а Y=\left(y_{1}, y_{2}, \ldots, y_{n}\right)$ - упорядоченный по неубыванию координат итоговый вектор допустимого решения задачи $A$, то для любого челого $l, 1 \leqslant k \leqslant n$, выполняется неравенство

$$
\sum_{i=1}^{k} x_{i} \geqslant \sum_{i=1}^{k} y_{i}
$$

Доказательство. Воспользуемся методом индукщии. При $k=1$ неравенство имеет вид $x_{1} \geqslant y_{1}$. Предположим противное, то есть пусть справедливо неравенство $x_{1}<y_{1}$. Рассмотрим $l$-срезки решений $X$ и $Y$ при $l=y_{1}$ и значение функщионала, определяющего оптимальность $A l$-задачи,

$$
\begin{aligned}
& \varphi(X[l])=\sum_{i=1}^{n} x[l]_{i}=\sum_{x_{i}<l} x_{i}+\sum_{a_{i} \geqslant l} a_{i}+\sum_{a_{i}<l, x_{i} \geqslant l} l, \\
& \varphi(Y[l])=\sum_{i=1}^{n} y[l]_{i}=\sum_{y_{i}<l} y_{i}+\sum_{a_{i} \geqslant l} a_{i}+\sum_{a_{i}<l, y_{i} \geqslant l} l .
\end{aligned}
$$


По числу слагаемых обе суммы совпадают. Вторые слагаемые в правых частях каждого из обоих выражений одинаковы и по значению и по количеству членов суммирования. Первое слагаемое в правой части второго выражения пустое, так как нет координат $y_{i}$ таких, что $y_{i}<l$, где $l=y_{1}$, так как вектор $Y$ упорядочен.

Учитывая упорядоченность вектора $Y$, предположение $x_{1}<y_{1}$ и равенство $l=y_{1}$, получаем неравенство

$$
\sum_{a_{i}<l, y_{i} \geqslant l} l>\sum_{x_{i}<l} x_{i}+\sum_{a_{i}<l, x_{i} \geqslant l} l,
$$

поскольку левая и правая его части совпадают по числу слагаемых. Отсюда следует неравенство

$$
\varphi(X[l])<\varphi(Y[l])
$$

чего быть не может, так как $X[l]$ - оптимальное решение $A l$-задачи. Поэтому $x_{1} \geqslant y_{1}$.

Предположим, что выполнены неравенств

$$
\sum_{i=1}^{1} x_{i} \geqslant \sum_{i=1}^{1} y_{i}, \quad \sum_{i=1}^{2} x_{i} \geqslant \sum_{i=1}^{2} y_{i}, \quad \ldots, \quad \sum_{i=1}^{k-1} x_{i} \geqslant \sum_{i=1}^{k-1} y_{i},
$$

и докажем утверждение леммы, состоящее в том, что

$$
\sum_{i=1}^{k} x_{i} \geqslant \sum_{i=1}^{k} y_{i}
$$

Если предположить противное, то есть, что

$$
\sum_{i=1}^{k} x_{i}<\sum_{i=1}^{k} y_{i}
$$

то учитывая (7), получаем неравенство $x_{k}<y_{k}$. Тогда при $l=y_{k}$ можно провести аналогичные приведенным выше доказательства того, что в этом случае срезка $X[l]$ теряет свойство оптимальности для $\mathrm{Al}$-задачи. Полученное противоречие доказывает лемму.

Докажем теорему об оптимальности критерия $L(X)$ в $A$-задаче.

Теорема 2. Если решение задачи $A$ оптимизирует критерий $L$, то оно оптимально и по любой другой симметрической норме.

Доказательство. Пусть $N$ - любая симметрическая норма, $X^{\prime}=\left(x_{1}^{\prime}, x_{2}^{\prime}, \ldots, x_{n}^{\prime}\right)$ - итоговый вектор $L$-оптимального решения $A$-задачи и $Y^{\prime}=\left(y_{1}^{\prime}, y_{2}^{\prime}, \ldots, y_{n}^{\prime}\right)$ - итоговый вектор допустимого решения $\boldsymbol{A}$-задачи. Требуется доказать неравенство

$$
N\left(X^{\prime}-S\right) \leqslant N\left(Y^{\prime}-S\right)
$$

где $S$ - вектор, каждая компонента которого равна среднему арифметическому числу работ $\bar{s}=s / n$. Вектор $S$ можно представить как $S=\bar{s} I$, где $I-$ вектор из единиц. Упорядочим элементы векторов $X^{\prime}$ и $Y^{\prime}$ по неубыванию, получив векторы

$$
X=\left(x_{1}, x_{2}, \ldots, x_{n}\right), \quad Y=\left(y_{1}, y_{2}, \ldots, y_{n}\right)
$$


соответственно, где

$$
x_{1} \leqslant x_{2} \leqslant \ldots \leqslant x_{n}, \quad y_{1} \leqslant y_{2} \leqslant \ldots \leqslant y_{n} .
$$

Тогда

$$
N\left(X^{\prime}-S\right)=N(X-S), \quad N\left(Y^{\prime}-S\right)=N(Y-S),
$$

так как $N$ - симметрическая норма. Заметим, что при этом полученные векторы могут не быть векторами допустимых решений.

Если вектор $Y$ представлен тем же набором элементов, что и вектор $X$, то значения нормы $N$ на обоих векторах $X-S$ и $Y-S$ одинаковы, то есть утверждение теоремы выполняется.

Пусть $X$ и $Y$ представлены различными наборами элементов. Построим последовательность $Y^{0}=Y, Y^{1}, \ldots, Y^{q}=X$ такую, что $N\left(Y^{i}-S\right) \geqslant N\left(Y^{i+1}-S\right)$ для любого $i \in\{0,1, \ldots, q-1\}$, следуюшим образом. Первый вектор $Y^{0}=Y=\left(y_{1}, y_{2}, \ldots, y_{n}\right)$ и для него согласно доказанной выше лемме выполняется неравенство

$$
\sum_{i=1}^{k} x_{i} \geqslant \sum_{i=1}^{k} y_{i}
$$

для любого $1 \leqslant k \leqslant n$. Дальнейшее построение опирается на это неравенство и не использует того факта, что вектор $Y$ является упорядоченным и получен перестановкой элементов допустимого вектора.

Пусть $x_{k}, y_{k}$ - первая пара неравных элементов, то есть $x_{i}=y_{i}$ при $i<k$, но $x_{k} \neq y_{k}$ (где $k$ может быть и единицей). Для $X$ и $Y$ выполняется свойство (6), поэтому $x_{k}>y_{k}$. Тогда найдется пара элементов, удовлетворяющая неравенству $x_{p}<y_{p}$ (сумма элементов каждого вектора постоянна и равна общему числу работ), где $p>k$, и пусть $p$ - наименьшее из всех возможных. Ясно, что $y_{k}<x_{k} \leqslant x_{p}<y_{p}$, и тогда $y_{p}-y_{k} \geqslant 2$.

Построим вектор

$$
Y^{1}=\left(y_{1}, \ldots, y_{k}+1, \ldots, y_{p}-1, \ldots, y_{n}\right),
$$

изменив в векторе $Y^{0}$ лишь два элемента $k$-й и $p$-й. Поменяв в векторе $Y^{0}$ два элемента $k$-й и $p$-й местами, получим вектор

$$
\bar{Y}^{0}=\left(y_{1}, \ldots, y_{p}, \ldots, y_{k}, \ldots, y_{n}\right) .
$$

Тогда $N\left(Y^{0}-S\right)=N\left(\bar{Y}^{0}-S\right)$, так как векторы $Y^{0}-S$ и $\bar{Y}^{0}-S$ различаются лишь порядком координат. Рассмотрим уравнение

$$
Y^{1}=\alpha Y^{0}+\beta \bar{Y}^{0} .
$$

Покоординатные уравнения, соответствуюшие $k$-й и $p$-й координате, имеют вид

$$
\begin{aligned}
& y_{k}^{1}=\alpha y_{k}+\beta y_{p}, \\
& y_{p}^{1}=\alpha y_{p}+\beta y_{k}
\end{aligned}
$$

или

$$
\begin{aligned}
y_{k}+1 & =\alpha y_{k}+\beta y_{p}, \\
y_{p}-1 & =\alpha y_{p}+\beta y_{k} .
\end{aligned}
$$


Так как $y_{p}-y_{k} \geqslant 2$, справедливы равенства

$$
\begin{aligned}
& \alpha=\frac{y_{k}^{2}+y_{k}-y_{p}^{2}+y_{p}}{y_{k}^{2}-y_{p}^{2}}=\frac{\left(y_{k}+y_{p}\right)\left(y_{k}-y_{p}+1\right)}{y_{k}^{2}-y_{p}^{2}} \geqslant 0, \\
& \beta=\frac{y_{k} y_{p}-y_{k}-y_{k} y_{p}-y_{p}}{y_{k}^{2}-y_{p}^{2}}=\frac{-\left(y_{k}+y_{p}\right)}{y_{k}^{2}-y_{p}^{2}} \geqslant 0,
\end{aligned}
$$

причем $\alpha+\beta=1$. При таких значениях $\alpha$ и $\beta$ остальные покоординатные уравнения удовлетворяются, так как соответствующие координаты всех трех векторов, участвующих в них, равны между собой. Тогда при найденных значениях $\alpha$ и $\beta$ выполняется равенство (8). Вычтя из обеих частей (8) вектор $S$, получим равенство

$$
Y^{1}-S=\alpha\left(Y^{0}-S\right)+\beta\left(\bar{Y}^{0}-S\right)
$$

откуда следует, что

$$
N\left(Y^{1}-S\right) \leqslant N\left(Y^{0}-S\right)
$$

Покажем, что построенный вектор $Y^{1}$ обладает свойством (6), то есть для любого $1 \leqslant t \leqslant n$ выполняется неравенство

$$
\sum_{i=1}^{t} x_{i} \geqslant \sum_{i=1}^{t} y_{i}^{1}
$$

Вектор $Y^{1}$ состоит из элементов

$$
Y^{1}=\left(y_{1}, \ldots, y_{k}+1, \ldots, y_{p}-1, \ldots, y_{n}\right)
$$

Так как координаты до $k$-й не изменены, для $t \leqslant k-1$ неравенство выполняется. Поскольку $x_{k}>y_{k}$, для $t=k$ неравенство также выполнено. Номер $p$ является наименьшим из всех возможных таких, что $x_{p}<y_{p}$. Поэтому для $i=k+1, \ldots, p-1$ выполняются неравенства $x_{i} \geqslant y_{i}$, что влечет выполнение неравенства для $t=k+1, \ldots, p-1$. Для остальных значений $t \geqslant p$ соответствующие суммы не изменились по сравнению с соответствуюшими суммами вектора $Y^{0}$. Поэтому свойство (6) для вектора $Y^{1}$ выполнено. Вектор $Y^{1}$ может не быть допустимым и даже упорядоченным, но для него выполнено свойство (6), которое было выполнено для вектора $Y^{0}$. Именно это свойство вектора $Y^{0}$ и позволило построить вектор $Y^{1}$. Поэтому можно взять вектор $Y^{1}$ вместо $Y^{0}$ в качестве начального и построить по тому же правилу вектор $Y^{2}$ такой, что $N\left(Y^{2}-S\right) \leqslant N\left(Y^{1}-S\right)$ и т. д. Построенная последовательность векторов $Y^{i}$ такова, что в каждом следующем векторе последовательности первая отличная от соответствующей координаты вектора $X$ координата приближается на единищу к ней. Приближение каждой очередной координаты продолжается до равенства с соответствующей координатой вектора $X$ и в следующем векторе построенной последовательности происходит приближение следующей координаты. Таким образом, последним вектором в последовательности выступает сам вектор $X$, что и влечет выполнение неравенства $N(X-S) \leqslant N(Y-S)$ и соответственно неравенства $N\left(X^{\prime}-S\right) \leqslant N\left(Y^{\prime}-S\right)$. Последнее неравенство означает, что вектор $X^{\prime}$, оптимизирующий норму $L$, оптимизирует и норму $N$, что и требовалось доказать. 


\section{Список литературы}

1. Кропанов В. А., Рублев В. С., Равномерное назначение работ минимальной стоимости. Дискретная математика (2001) 13, №4, 144-156.

2. Рублев В. С., Задача о равномерном распределении работ. Деп. ВИНИТИ №611-В87 26.01.87.

3. Рублев В. С., Чаплыгина Н. Б., Расширение задачи о назначениях. Моделирование и анализ информачионных систем (2002) 9, №2, 3-11.

Статья поступила 31.03.2005. 